\title{
Sexual function following pelvic fracture urethral injury and posterior urethroplasty
}

\author{
Andrew Mazzone ${ }^{1 \#}$, Ross Anderson ${ }^{2 \#}$, Bryan B. Voelzke ${ }^{3}$, Alex J. Vanni ${ }^{4}$, Sean P. Elliott ${ }^{5}$, \\ Benjamin N. Breyer ${ }^{6}$, Bradley A. Erickson ${ }^{7}$, Jill Buckley ${ }^{8}$, Jeremy Myers ${ }^{1}$; for the Trauma Urologic \\ Research Network of Surgeons
}

${ }^{1}$ University of Utah, Salt Lake City, UT, USA; ${ }^{2}$ Urology Nevada, Reno, NV, USA; ${ }^{3}$ Spokane Regional Health District, Spokane, WA, USA; ${ }^{4}$ Lahey Hospital and Medical Center Burlington, Burlington, MA, USA; ${ }^{5}$ University of Minnesota, Minneapolis, MN, USA; ${ }^{6}$ University of California San Francisco, San Francisco, CA, USA; ${ }^{7}$ University of Iowa, Iowa City, IA, USA; ${ }^{8}$ University of California San Diego, San Diego, CA, USA

Contributions: (I) Conception and design: A Mazzone, R Anderson, J Myers; (II) Administrative support: All authors; (III) Provision of study materials or patients: All authors; (IV) Collection and assembly of data: A Mazzone, R Anderson, J Myers; (V) Data analysis and interpretation: All authors; (VI) Manuscript writing: All authors; (VII) Final approval of manuscript: All authors.

"These authors contributed equally to this work and are co-first authors.

Correspondence to: Andrew Mazzone, MD. University of Utah, Salt Lake City, UT, USA. Email: Andrew.Mazzone@hsc.utah.edu.

Background: To evaluate erectile and sexual function after pelvic fracture urethral injury (PFUI) by performing a retrospective review of a large multi-center database. We hypothesized that most men will have erectile dysfunction (ED) and poor sexual function following PFUI, which will remain after posterior urethroplasty.

Methods: Using the Trauma and Urologic Reconstructive Networks of Surgeons (TURNS) database, we identified PFUI patients undergoing posterior urethroplasty. We excluded patients with incomplete demographic, surgical and/or questionnaire data. Sexual Health Inventory of Men (SHIM), Male Sexual Health Questionnaire (MSHQ), and subjective changes in penile curvature were collected before urethroplasty surgery and at follow-up. We performed descriptive statistics for erectile and ejaculatory function using STATA v12.

Results: We identified 92 men meeting inclusion criteria; median age was 41.7 years and BMI was 26.5. The mechanism of injury was blunt in all patients, and average distraction defect length was $2.3 \mathrm{~cm}(\mathrm{SD} 1.0 \mathrm{~cm})$. In the 38 patients who completed both pre and post-operative SHIM questionnaires, the mean SHIM score was 10.5 (SD 7.0), with 63\% having severe ED (SHIM <12). The median follow-up was 5.6 months and the mean post-operative SHIM was 9.3 (SD 6.5), with 68\% having severe ED. The mean change in SHIM score was -1.18 (SD 6.29) with 6 (16\%) patients reporting de novo $\mathrm{ED}$ ( $\geq 5$ point decrease in score). Of the men with pre-operative MSHQ data, 46/74 (62.1\%) had difficulty with ejaculation, 25/35 (71\%) had change in penile length, and 6/33 (18\%) reported penile curvature. In men with post-operative MSHQ, 19/44 (43\%) expressed difficulty with ejaculation, 23/32 (72\%) had change in penile length, and 9/33 (27\%) reported penile curvature.

Conclusions: There is a high rate of severe ED, both following PFUI and remaining after posterior urethroplasty. Additionally, rates of ejaculatory difficulty and patient perceived changes in penile length and curvature underscore the complex nature of the impact of these injuries on sexual function beyond simple erectile function.

Keywords: Erectile dysfunction (ED); pelvic fracture; sexual function; trauma; urethroplasty

Submitted Sep 27, 2020. Accepted for publication Mar 31, 2021.

doi: $10.21037 /$ tau-20-1287

View this article at: http://dx.doi.org/10.21037/tau-20-1287 


\section{Introduction}

Pelvic fractures make up approximately $3 \%$ of all skeletal injuries, and the incidence among admitted trauma patients ranges from $8-9 \%(1-3)$. Furthermore, $2 \%$ of men with traumatic pelvic fracture have a concurrent pelvic fracture urethral injury (PFUI) $(4,5)$. Improved trauma care and genitourinary reconstruction has led to improved survival and functional status in these patients (5-7). However these injuries can be devastating for men psychologically due to profound impact on their sexual function.

The rates of erectile dysfunction (ED) after pelvic fracture alone range from $5-28 \%$, while the incidence of $\mathrm{ED}$ after PFUI is much higher, and varies widely in the literature from $26-76 \%$ (8-12). Urethral repair with posterior urethroplasty has demonstrated excellent long-term success rates, but long term erectile and sexual function assessment in these patients has been limited by the way the studies report ED, as mostly binary variables (yes vs. no) $(6,13)$. All previously published rates of ED are from small singlecenter retrospective studies (14-18). These studies were also mostly limited by the lack of validated metrics, with only one study that used the International Index of Erectile Function (IIEF-5) for classifying ED (19).

Using the Trauma and Urologic Reconstructive Networks of Surgeons (TURNS) database, we sought to quantify erectile and sexual function using two validated questionnaires, the Sexual Health Inventory of Men (SHIM), selected questions from the Male Sexual Health Questionnaire (MSHQ), as well as non-validated questions about penile length and curvature. Also, we sought to accurately define changes in erectile and sexual function that occur following posterior urethroplasty. We hypothesize that the majority of men will have ED and poor sexual function following their injuries and there will be minimal change after posterior urethroplasty. We present the following article in accordance with the STROBE reporting checklist (available at http://dx.doi.org/10.21037/tau-20-1287).

\section{Methods}

We utilized the TURNS urethroplasty database to identify patients with PFUI who underwent posterior urethroplasty. TURNS is a multi-institutional group focused on urologic trauma and reconstruction, made up of 13 urologic surgeon members, who have an established prospectively collected database of urethroplasty patients (20). The TURNS group has published their long-term voiding outcomes in 122 patients that underwent posterior urethroplasty following
PFUI, with a success rate of $91 \%$ (6). We reviewed the database of patients from 2009 to 2017, and identified 92 of the 122 patients who completed erectile and sexual function questionnaires either before, after, or both before and after urethroplasty. We excluded 30 patients that had no pre or post-urethroplasty SHIM/MSHQ data. We restricted our SHIM analysis to 38 patients with both pre and post urethroplasty data. With the MSHQ data this was not possible due to lack of patients with both pre and post urethroplasty data and instead the group responses were compared between pre and post urethroplasty, and change was not assessed in patients with complete pre and post urethroplasty data. Injuries were classified as blunt or penetrating. Pre-injury erectile function was not available.

All patients were managed with either endoscopic primary urethral realignment or suprapubic catheter (SPC), followed by delayed posterior urethroplasty repair. Adjunct maneuvers during posterior urethroplasty to achieve a tension free urethral anastomosis were noted and included: (I) abdominal-perineal approach; (II) corporal re-routing; (III) pubic symphysis resection; (IV) corporal splitting; (V) partial prostatectomy. The SHIM has been validated to measure ED in multiple settings and was administered along with the MSHQ, which assesses ejaculatory and other aspects of sexual function $(21,22)$. SHIM \& MSHQ data were obtained pre- and post-operatively at each followup. SHIM scores range from 1-25, with lower numbers signifying worse ED. To simplify our SHIM categories, the SHIM data were further stratified by the severity of ED: minimal $>21$, moderate 12-20 (combines both "mild and mild to moderate"), and severe $<12$ (combines both "moderate and severe") (23). The MSHQ data obtained was limited to the following questions: how often have you been able to ejaculate, how would you rate the strength of ejaculate, how would you rate the amount of ejaculate, and the bothersome score for ejaculate. Therefore, we did not include a MSHQ composite score. Sexual function was further evaluated using non-validated questions of patientperceived changes in penile length and curvature both pre- and post-operatively. The study was conducted in accordance with the Declaration of Helsinki (as revised in 2013). The study was approved by the institutional review board of the University of Utah (IRB_00047027) and informed consent was taken from all the patients.

\section{Statistical analysis}

Descriptive statistics were performed using STATA v12. 
Table 1 Patient demographics and injury characteristics (N=92)

\begin{tabular}{lc}
\hline Patient variables & Total (\%) \\
\hline Age (years), median (IQR) & $41.7(30.0-53.9)$ \\
BMI, median (IQR) & $26.5(22.9-31.1)$ \\
Stricture length (cm), median (IQR) & 2 [2-3] \\
Need for embolization & 3 \\
Unilateral & 5 \\
Bilateral & \\
Medical co-morbidities & 28 \\
Current or former smokers & 5 \\
Diabetic & 14 \\
Hypertension & 10 \\
Hyperlipidemia & 0 \\
PVD or CAD & 13 \\
Adjunct procedures & \\
Abdominal perineal approach & 5 \\
Corporal splitting & 35 \\
Corporal re-routing & 1 \\
Pubic symphysis resection & \\
Partial prostatectomy & \\
\hline BM, body mass index; & \\
\hline
\end{tabular}

BMI, body mass index; PVD, peripheral vascular disease; CAD, coronary artery disease.

Two-sided $t$-tests were used to assess association of post-op ED with the following potential variables: initial catheter management, pelvic angioembolization (coil or gelfoam) during initial trauma management. Two sample rank-sum (Mann-Whitney) tests and chi-square tests were used to compare severe ED rates with adjunctive maneuvers at the time of posterior urethroplasty.

\section{Results}

\section{Patient demographics and injury characteristics}

In the 92 patients with questionnaire data available, the median age at injury was 41.7 years (IQR, 30-53.9 years) and median BMI was 26.5 (IQR, 22.9-31.1); mechanism of injury was blunt injury in all patients. The median distraction defect length was $2 \mathrm{~cm}$ (IQR, 2-3 cm). The median followup was 5.6 months (IQR, 4.1-7.8 months) after urethroplasty. Eight patients required angioembolization to control pelvic bleeding. Suprapubic catheter management just prior to urethroplasty was present in $62 \%$ of patients. Twenty-eight patients $(30 \%)$ reported current or former smoking status (Table 1).

\section{Patient reported sexual function (SHIM)}

Of the 92 patients identified in the database, 74 (80.4\%) completed pre-operative SHIM questionnaires; 44 (47.8\% of the 92) completed post-operative SHIM questionnaires. There were 38 patients who completed both a pre and postoperative SHIM questionnaire, and were the focus of our $\mathrm{ED}$ analysis. The mean pre-operative SHIM score in the cohort of 38 patients was 10.5 (SD 7.0), which decreased (worsened) to 9.3 (SD 6.5) post-operatively. The rate of Severe ED (SHIM <12) was reported in 63\% (24 of 38) preoperatively and was not significantly different from the $68 \%$ (26 of 38) post-operatively (Figure 1). The mean change in SHIM score was -1.18 (SD 6.29, not statistically significant) with $6(16 \%)$ patients reporting de novo ED ( $\geq 5$-point decrease in score).

\section{Patient reported sexual function (MSHQ, penile length and curvature)}

There were 74 patients with MHSQ scores either pre or post urethroplasty. There were 46/74 (62.1\%) patients that expressed difficulty with ejaculation (either unable to ejaculate or only able to ejaculate less than half of the time), and $51 / 74(68.9 \%)$ that reported bother by their ejaculatory difficulties after PFUI. There were 25/35 (71\%) men with reported change in penile length, and 6/33 (18\%) reported penile curvature. In men with post-operative MSHQ, 19/44 (43\%) expressed difficulty with ejaculation, 23/32 (72\%) had change in penile length, and 9/33 (27\%) reported penile curvature (Figure 2).

\section{Adjunctive maneuvers}

There were $45(48.9 \%)$ patients that needed one or more of the following adjunct maneuvers at the time of surgery, including: corporal splitting $(\mathrm{N}=35)$, abdominalperineal approach $(\mathrm{N}=5)$, corporal re-routing $(\mathrm{N}=1)$, pubic symphysis resection $(\mathrm{n}=10)$, or partial prostatectomy $(\mathrm{N}=13)$ (Table 1). There was no significant difference, but there was a trend towards worse post-operative SHIM score when we compared those patients with or without adjunct maneuvers 


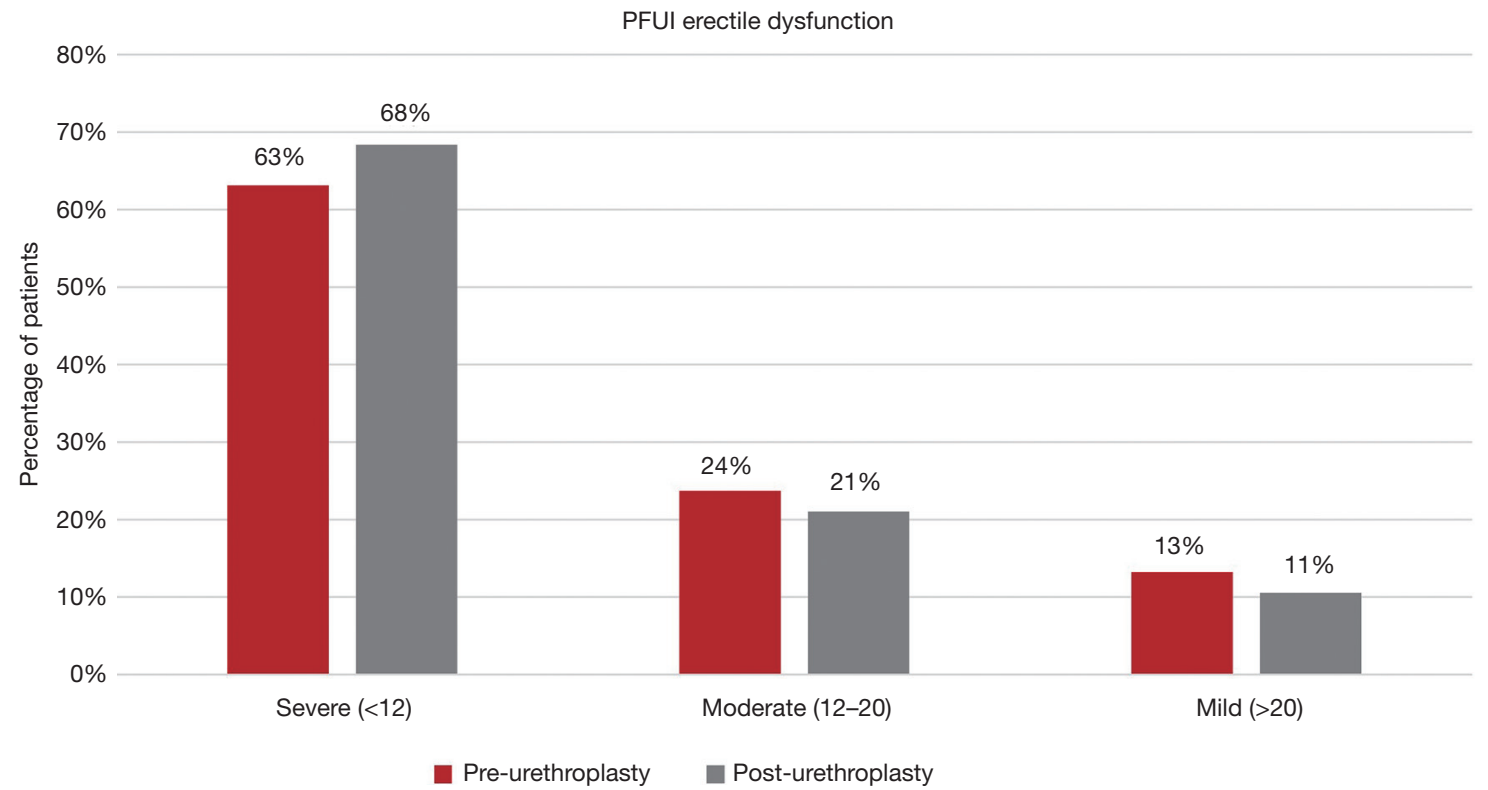

Figure 1 SHIM scores before and after urethroplasty. Only includes the 38 patients who completed both the pre and post questionnaires. SHIM, sexual health inventory of men.

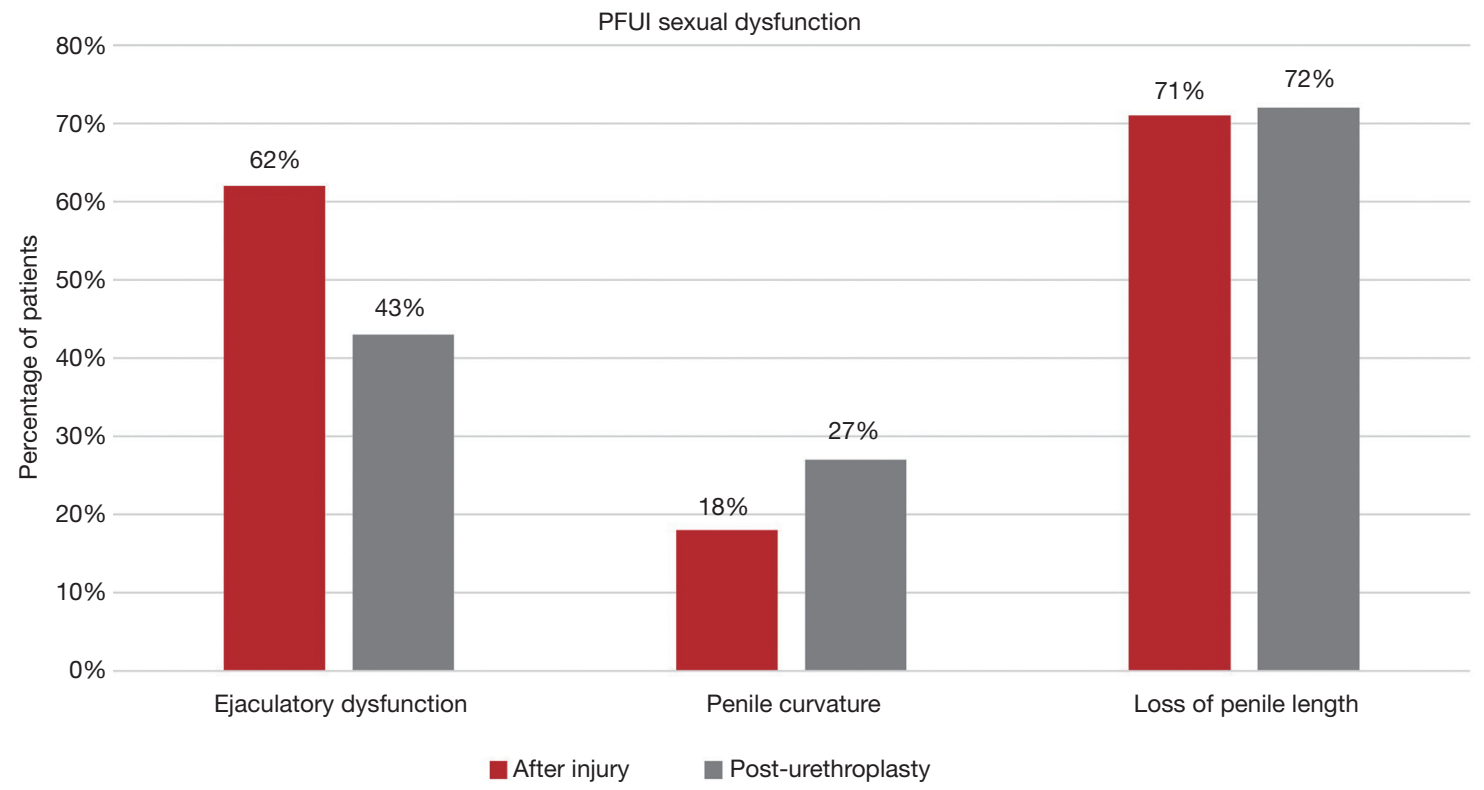

Figure 2 Sexual dysfunction rates after injury and post-urethroplasty.

at the time of urethroplasty (mean SHIM 7.6 vs. 10.8, $\mathrm{P}=0.19$ ). When analyzing the subgroup of patients who underwent adjunctive maneuvers and had completed postoperative SHIM data, we found that adjunct maneuvers were more likely to have severe $\mathrm{ED}(\mathrm{P}=0.043)$, compared to those without any adjunct maneuvers (Table 2). Suprapubic tube management pre-operatively was also not associated with a significant difference in post-operative SHIM score (SPT mean SHIM 9.5 vs. non-SPT mean $10.4, \mathrm{P}=0.72$ ). Four patients underwent microvascular revascularization surgery, 
Table 2 Erectile function in patients who underwent adjunct surgical maneuvers and completed post op SHIM (N=43)

\begin{tabular}{lcc}
\hline Adjuvant maneuvers & SHIM $<12(\mathrm{~N}=30)$ & SHIM $>12(\mathrm{~N}=13)$ \\
\hline Adjunct procedures at time of surgery (total patients) & 17 & 3 \\
Abdominal perineal approach & 0 & 1 \\
Corporal splitting & 14 & 3 \\
Corporal re-routing & 1 & 0 \\
Pubic symphysis resection & 3 & 1 \\
Partial prostatectomy & 6 & 0 \\
\hline
\end{tabular}

*, a single patient may have 1 or more adjunct procedures; ${ }^{*}$, of the patients with post-operative SHIM scores, having one or more adjunct procedures was associated with higher risk of severe ED (SHIM <12). SHIM, Sexual Health Inventory of Men; ED, erectile dysfunction.

with two patients requiring bilateral revascularization. Prior to urethroplasty 3 of 4 patients had a SHIM score of 5, and one patient reported SHIM 12. Unfortunately, 3 of 4 patients did not have follow up SHIM data, and the one patient with post-urethroplasty SHIM remained at 5 (most severe ED). Pelvic angioembolization at the time of injury, either uni- or bilaterally, did not significantly impact the mean SHIM score with all groups averaging SHIM scores in the severe range, and this was similar at both time points $(\mathrm{P}=0.99)$.

\section{Discussion}

Using the TURNS multi-center, prospectively collected, database we identified patients with PFUI undergoing posterior urethroplasty and found high rates of severe erectile and sexual dysfunction. In our series, $63 \%$ of patients had severe ED after PFUI, and remained high at $68 \%$ following definitive surgical repair. While success rates for continence has been well studied, previous reports in these studies did not use validated metrics when reporting ED rates. We also report important sexual dysfunction metrics using the MSQH and non-validated questionnaires, which demonstrated PFUI patients have high rates of ejaculatory dysfunction, perceived loss of penile length, and new and bothersome penile curvature.

The mechanism of ED following PFUI is focused on the direct injury that both lacerates and often avulses the posterior urethra, while simultaneously injuring the penile nerves and arteries as they traverse the pelvic floor posterior to the apex of the prostate prior to entering the penis. There is debate and limited literature as to the contribution of neurogenic injury compared to direct vascular injury, but more evidence suggests that neurogenic
ED is the more common etiology. One study supporting the neurogenic injury theory found that 24 of 27 (89\%) of impotent men following PFUI responded to intracavernosal vasoactive medications (24). In a separate study, corpora cavernosal electromyogram in 5 of 8 men with PFUI and ED were abnormal despite normal vascular flow on duplex ultrasound (25). Arguments for vasculogenic ED etiology from PFUI are based on arteriographic studies that report occlusion rates as high as $92 \%$ of either internal pudendal or internal penile artery (26). Although controversial, revascularization of the internal pudendal arteries was reported to successfully treat ED in $82 \%$ of men with bilateral arterial occlusion following PFUI (27). In our study, only four patients (4.3\%) underwent microvascular revascularization surgery for ED. Furthermore, the severity of ED was similar for men who required angioembolization during their initial trauma management compared to men without angioembolization. Both findings from our study would support the role of neurogenic ED for men with PFUI, but our study is likely underpowered to definitively describe the etiology of ED.

There is limited data that helps identify which patients will develop sexual dysfunction or ED following PFUI. Predictors of ED on multivariable analysis in pediatric patients, reported by Koraitim et al., included urethral gap distance $>2.5 \mathrm{~cm}$ and lateral displacement of the prostate (15). Another study by Johnsen et al., found that only high grade urethral injury predicted ED (28). Our study showed mean gap distance of $2.3 \mathrm{~cm}$, and our data did not include the direction of displacement or the American Association of Surgery for Trauma (AAST) Urethral Trauma Grading Scale.

Initial bladder management with either SPC $v s$. primary endoscopic realignment in the setting of PFUI is hotly 
debated, and is currently under study by the Multiinstitutional Genitourinary Study Group supported by the AAST (29). One of questions in this study examines the impact of bladder management on long-term sexual function. Previous studies, including a meta-analysis have failed to show a significant difference in ED rates based on bladder management after PFUI, although SPC has trended towards higher rates of $\mathrm{ED}(30,31)$. One of the major limitations of the previous cohort studies and the meta-analysis was the lack of validated ED questionnaire data. In addition, those men that were treated with urethral realignment may have less severe injuries, creating selection bias and the appearance of better sexual function that is not actually related to their bladder management. In our cohort of men, SPC did not impact the severity of ED based on SHIM scores.

Many studies have attempted to determine the impact that posterior urethroplasty in PFUI patients has on erectile function. In the literature, the rate of improved erections following posterior urethroplasty ranged between 9-66\%, and the rate of de novo ED ranged between $3 \%$ and $7 \%$ after PFUI $(11,14,17,32,33)$. The largest series of posterior urethroplasty reported, from Joshi et al., described a $56 \%$ rate of ED pre- vs. $63 \%$ post-operatively, which was very similar to our findings (68\% post-operative severe ED rate) (13). In our study, ED was worsened in $16 \%$ of patients after urethroplasty. This finding was also reported in the one other study that used a validated questionnaire, the IIEF, where patients diagnosed with neurogenic ED experienced worsening ED postoperatively (IIEF 15 pre- vs. 10 postoperatively) (19).

Sexual dysfunction following PFUI and posterior urethroplasty has even less available literature than ED. In one study, among 277 pelvic fracture patients surveyed, $46 \%$ of men reported sexual dysfunction; however, the definitions of sexual dysfunction were not well explained (34). In our study, $70 \%$ of patients following their injury experienced perceived penile length loss, and this proportion did not change after urethroplasty. Similarly, about one quarter of men reported penile curvature, both before and after surgery. If the loss of length or curvature was only seen post-operatively it would be simple to account for this based on the anastomotic nature of the repair, but this was present pre-operatively. Previous literature has described fibrosis of the corpora cavernosa associated with neurovascular injury after radical prostatectomy which can lead to penile shortening and ED (33). The mechanism of penile shortening after PFUI and posterior urethroplasty may be very similar. One study reported that patients with PFUI and posterior urethroplasty had decreased penile girth and length compared to traumatic anterior urethral stricture and subsequent urethroplasty (18). The one sexual function parameter that improved significantly in our study was ejaculatory function, with only $40 \%$ of men with ejaculatory dysfunction after posterior urethroplasty compared to $60 \%$ after their injury. Pre-urethroplasty the mechanism could be explained by the complete obliteration of the posterior urethra. After successful and patent anastomosis, the remaining ejaculatory dysfunction may be due to neurogenic dysfunction of the hypogastric somatic nerves necessary for antegrade emission or due to posturethroplasty bulbospongiosus muscle dysfunction. The exact mechanism of ejaculatory dysfunction after anterior urethroplasty has remained elusive.

This study has many limitations. Most notably, patient follow-up, survey rates, and the multiple different surgeons can introduce potential uncontrolled biases. With multiple surgeons in the group, individual techniques may not fully be quantified even with this advanced database. Due to the rare nature and complexity of this injury, many of the patients travel great distances to be treated by the TURNS surgeons, and subsequently follow up with local urologists. This contributed to our moderate follow-up time. With a median follow-up time of 6 months, if we extrapolate data from prostatectomy, then it is likely that erectile and sexual function will continue to improve up to $12-18$ months following the injury. Previous researchers have proposed that tissue edema, swelling, and inflammation are all causes of ED that may explain the transient deterioration of erectile function after surgery, and which may continue to improve beyond 6 months (35). An insufficient number of patients underwent penile revascularization prior to posterior urethroplasty and for this reason a subset analysis of the effects of revascularization on erectile and sexual function was not possible. We acknowledge that this study would be improved if all patients had complete sexual and erectile function questionnaires both pre- and post-urethroplasty. With this data it may be possible to see differences in functional outcomes based on adjunct maneuvers at that time of surgery, but there are many challenges obtaining sexual and erectile function data in this population.

While the majority of men have severe ED after PFUI, identifying the rare patients with preserved erectile and sexual function following PFUI is imperative for careful surgical planning and pre-operative counseling. We anxiously await results from the AAST randomized clinic 
trial for initial bladder management in PFUI, and look forward to any recommendations to improve the significant sexual and erectile morbidity experienced by these patients. The management of PFUI patients may benefit from a multi-disciplinary approach, including early referral to mental health. We also advocate for referral to our men's health clinic for a penile rehabilitation program to help address and mitigate the effects of PFUI on erectile and sexual function.

\section{Conclusions}

ED is common after PFUI, with larger defects correlating with worse function. Urethroplasty appears to negatively affect erectile function in select patients. Other aspects of sexual function, including penile length, curvature and ejaculatory function, are also common, underscoring the complex nature of the impact of these injuries on sexual function beyond simple erectile function, and may serve to guide future management strategies in this patient population.

\section{Acknowledgments}

Funding: None.

\section{Footnote}

Reporting Checklist: The authors have completed the STROBE reporting checklist. Available at http://dx.doi. org/10.21037/tau-20-1287

Data Sharing Statement: available at http://dx.doi. org/10.21037/tau-20-1287

Conflicts of Interest: All authors have completed the ICMJE uniform disclosure form (available at http://dx.doi. org/10.21037/tau-20-1287). AJV reports personal fees from Expert Testimonial, serving as expert testimony and as a consultant for Orchestra Biomed, both outside the submitted work. SPE reports grant funding for Urotronic and is a consultant for Boston Scientific and a shareholder of Percuvision, all outside the submitted work. BAE serves as an unpaid editorial board member of Translational Andrology and Urology from Jan 2019 to Dec 2021. JM serves as an unpaid editorial board member of Translational Andrology and Urology from Aug 2020 to Jul 2022. The other authors have no conflicts of interest to declare.
Ethical Statement: The authors are accountable for all aspects of the work in ensuring that questions related to the accuracy or integrity of any part of the work are appropriately investigated and resolved. The study was conducted in accordance with the Declaration of Helsinki (as revised in 2013). The study was approved by the institutional review board of the University of Utah (IRB_00047027) and informed consent was taken from all the patients.

Open Access Statement: This is an Open Access article distributed in accordance with the Creative Commons Attribution-NonCommercial-NoDerivs 4.0 International License (CC BY-NC-ND 4.0), which permits the noncommercial replication and distribution of the article with the strict proviso that no changes or edits are made and the original work is properly cited (including links to both the formal publication through the relevant DOI and the license). See: https://creativecommons.org/licenses/by-nc-nd/4.0/.

\section{References}

1. Grotz MRW, Allami MK, Harwood P, et al. Open pelvic fractures: epidemiology, current concepts of management and outcome. Injury 2005;36:1-13.

2. Sathy AK, Starr AJ, Smith WR, et al. The Effect of Pelvic Fracture on Mortality After Trauma: An Analysis of 63,000 Trauma Patients. J Bone Joint Surg Am 2009;91:2803-10.

3. Schulman JE, O’Toole RV, Castillo RC, et al. Pelvic Ring Fractures Are an Independent Risk Factor for Death After Blunt Trauma. J Trauma 2010;68:930-4.

4. Demetriades D, Karaiskakis M, Toutouzas K, et al. Pelvic fractures: epidemiology and predictors of associated abdominal injuries and outcomes1 1No competing interests declared. J Am Coll Surg 2002;195:1-10.

5. Johnsen NV, Dmochowski RR, Young JB, et al. Epidemiology of Blunt Lower Urinary Tract Trauma With and Without Pelvic Fracture. Urology 2017;102:234-9.

6. Johnsen NV, Moses RA, Elliott SP, et al. Multicenter analysis of posterior urethroplasty complexity and outcomes following pelvic fracture urethral injury. World J Urol 2020;38:1073-9.

7. Kulkarni SB, Joshi PM, Hunter C, et al. Complex posterior urethral injury. Arab J Urol 2015;13:43-52.

8. Mundy AR. Pelvic fracture injuries of the posterior urethra. World J Urol 1999;17:90-5.

9. King J. Impotence after fractures of the pelvis. J Bone Joint Surg Am 1975;57:1107-9. 
10. Shenfeld OZ, Kiselgorf D, Gofrit ON, et al. The incidence and causes of erectile dysfunction after pelvic fractures associated with posterior urethral disruption. J Urol 2003;169:2173-6.

11. Flynn BJ, Delvecchio FC, Webster GD. Perineal Repair of Pelvic Fracture Urethral Distraction Defects: Experience in 120 Patients During the Last 10 Years. J Urol 2003;170:1877-80.

12. Blaschko SD, Sanford MT, Schlomer BJ, et al. The incidence of erectile dysfunction after pelvic fracture urethral injury: A systematic review and meta-analysis. Arab J Urol 2015;13:68-74.

13. Joshi PM, Kulkarni SB. Management of pelvic fracture urethral injuries in the developing world. World J Urol 2020;38:3027-34.

14. Koraitim MM. On the art of anastomotic posterior urethroplasty: a 27-year experience. J Urol 2005;173:135-9.

15. Koraitim MM. Predicting risk of erectile dysfunction after pelvic fracture urethral injury in children. J Urol 2014;192:519-23.

16. Corriere JN Jr, Sandler CM. Management of the ruptured bladder: seven years of experience with 111 cases. J Trauma 1986;26:830-3.

17. Morey AF, McAninch JW. Reconstruction of posterior urethral disruption injuries: outcome analysis in 82 patients. J Urol 1997;157:506-10.

18. Feng C, Xu YM, Yu JJ, et al. Risk factors for erectile dysfunction in patients with urethral strictures secondary to blunt trauma. J Sex Med 2008;5:2656-61.

19. Tang CY, Fu Q, Cui RJ, et al. Erectile dysfunction in patients with traumatic urethral strictures treated with anastomotic urethroplasty: a single-factor analysis. Can J Urol 2012;19:6548-53.

20. Cotter KJ, Hahn AE, Voelzke BB, et al. Trends in Urethral Stricture Disease Etiology and Urethroplasty Technique From a Multi-institutional Surgical Outcomes Research Group. Urology 2019;130:167-74.

21. Cappelleri JC, Rosen RC, Smith MD, et al. Diagnostic evaluation of the erectile function domain of the international index of erectile function. Urology 1999;54:346-51.

22. Rosen RC, Catania J, Pollack L, et al. Male Sexual Health Questionnaire (MSHQ): Scale development and psychometric validation. Urology 2004;64:777-82.

23. Cappelleri JC, Rosen RC. The Sexual Health Inventory for Men (SHIM): a 5-year review of research and clinical experience. Int J Impot Res 2005;17:307-19.

24. Mark SD, Keane TE, Vandemark RM, et al. Impotence following pelvic fracture urethral injury: incidence, aetiology and management. Br J Urol 1995;75:62-4.

25. Machtens S, Gansslen A, Pohlemann T, et al. Erectile dysfunction in relation to traumatic pelvic injuries or pelvic fractures. BJU Int 2001;87:441-8.

26. Levine FJ, Greenfield AJ, Goldstein I. Arteriographically Determined Occlusive Disease within the HypogastricCavernous Bed in Impotent Patients Following Blunt Perineal and Pelvic Trauma. J Urol 1990;144:1147-53.

27. Zuckerman JM, McCammon KA, Tisdale BE, et al. Outcome of penile revascularization for arteriogenic erectile dysfunction after pelvic fracture urethral injuries. Urology 2012;80:1369-73.

28. Johnsen NV, Kaufman MR, Dmochowski RR, et al. Erectile Dysfunction Following Pelvic Fracture Urethral Injury. Sex Med Rev 2018;6:114-23.

29. Moses RA, Selph JP, Voelzke BB, et al. An American Association for the Surgery of Trauma (AAST) prospective multi-center research protocol: outcomes of urethral realignment versus suprapubic cystostomy after pelvic fracture urethral injury. Transl Androl Urol 2018;7:512-20.

30. Johnsen NV, Dmochowski RR, Mock S, et al. Primary Endoscopic Realignment of Urethral Disruption Injuries-A Double-Edged Sword? J Urol 2015;194:1022-6.

31. Barrett K, Braga LH, Farrokhyar F, et al. Primary realignment vs suprapubic cystostomy for the management of pelvic fracture-associated urethral injuries: a systematic review and meta-analysis. Urology 2014;83:924-9.

32. Myers JB, McAninch JW. Management of posterior urethral disruption injuries. Nat Clin Pract Urol 2009;6:154-63.

33. Corriere JN. 1-stage delayed bulboprostatic anastomotic repair of posterior urethral rupture: 60 patients with 1-year follow up. J Urol 2001;165:404-7.

34. Johnsen NV, Lang J, Wessells H, et al. Barriers to Care of Sexual Health Concerns in Men Following Traumatic Pelvic Fractures. J Sex Med 2019;16:1557-66.

35. Sangkum P, Levy J, Yafi FA, et al. Erectile dysfunction in urethral stricture and pelvic fracture urethral injury patients: diagnosis, treatment, and outcomes. Andrology 2015;3:443-9.

Cite this article as: Mazzone A, Anderson R, Voelzke BB, Vanni AJ, Elliott SP, Breyer BN, Erickson BA, Buckley J, Myers J; for the Trauma Urologic Research Network of Surgeons. Sexual function following pelvic fracture urethral injury and posterior urethroplasty. Transl Androl Urol 2021;10(5):2043-2050. doi: $10.21037 /$ tau-20-1287 